\section{ON "TENDON-REFLEX" AS AN AID TO DIAGNOSIS IN DISEASES OF THE SPINAL CORD.'}

By THOMAS BUZZARD, M.D., F.R.C.P.,

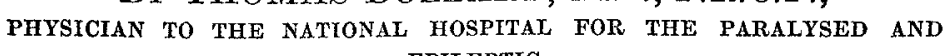
EPILEPTIC.

(Concluded from p. 84.)

WE have now to suppose the case of the patellar tendonreflex being absent in a patient who is either entirely confined to his bed or only able to walk with some difficulty. The quadriceps extensor is found more or less wasted, we will say, its response to induced currents being either below the normal standard of excitability or lost altogether. In the latter case there will be inability to contract the muscle by voluntary effort. Such a condition necessarily indicates that the muscle is cut off to some extent by some means or other from the trophic influence of the spinal cord. The seat of this influence is known to be in the large ganglion-cells which lie in the anterior horn. Now, it will be seen, if we look at the diagram (Fig. 3), that the interruption in this
FIG. 3.

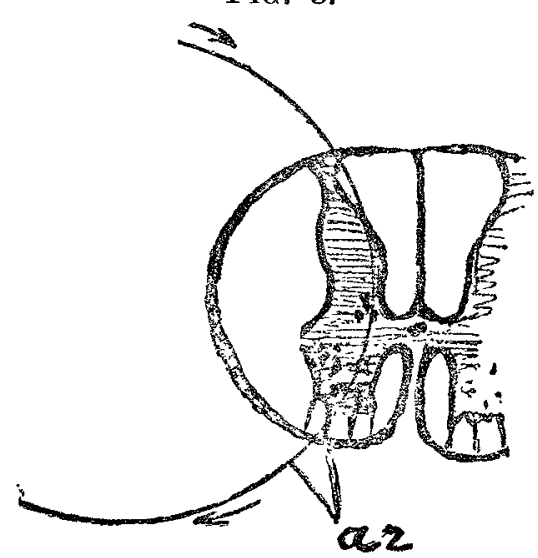
case may exist either in the anterior root outside the cord, the short portion of it within the cord, or in the anterior horn of grey matter itself. As regards the second of these possibilities, nothing is known for certain that will enable us to localise lesion in that particular portion of the anterior root. For the rest, absence or marked decrease of reflex, along with muscular wasting and loss of faradaic excitability, may be caused by neuritis of $a$ mixed spinal nerve, or it may result from spinal meningitis, involving the anterior roots either alone or in conjunction with the posterior roots. The precise cause for the interruption in such a case can only be determined by the consideration of collateral circumstances, into which I need not enter at length. It may be remarked, however, that if the symptoms described are accompanied by pain and tenderness in the nerve trunk, and some cutaneous insensibility, this sensory disturbance makes it evident that the cause of interruption is in the mixed nerve (in which the motor and sensory roots are combined) and not in the anterior (motor) root alone. I have recorded cases of neuritis in which marked decrease of tendon-reflex occurred in these circumstances. ${ }^{2}$ All that is sure from percussion of the tendon and examination of the muscle is that a break exists somewhere on the motor side of the nervous arc. Nor may there be anything in the condition of the muscle in such a case to distinguish it from that which obtains when the break in the nervous arc is brought about by atrophy of the large ganglion-cells in the anterior horn. In the rough diagram (Fig. 4) it is seen that the line, indicating the nervous arc, passes through an anterior horn which is shrunk and atrophied, and in which there is but little trace of ganglion-cells to be observed, in striking contrast to the number and size of those in the anterior horn of the other side. In this shrunken anterior horn, then, the

I In the preceding portion of this paper, published last week, I referred to a case of hemi-paraplegia under the care of Dr. Ramskill, and after mentioning that there was paralysis with extensive wasting of the left in the right lower extremity, I went on to state that the patellar in the right lower extremity, I went on to state that the patellar in the right limb to the fact that the lesion of the cord, although pro in the right limb to the fact that the lesion of the cord, although pro bably to a large extent confined to the left segment, was not absulutely
so. I thought that it encroached a little upon the right half of the cord, far enough to interfere with the integrity of the grey matter to a certain extent without affecting, however, the anterior horn. I ought to have mentioned that this view, which appears to me to be necessary in order to explain the break in the nervous arc subserving patellar tendon-reflex in the raght limb, is supported by the existence of a patch of cntaneous anesthesia, a bout eight inches by four, in the neighbourhood of the left great trochanter.

2 THE haNCET, April, 1879 "fault" occurs which causes absence of tendon-reflex. Now atrophy of the ganglion-cells of the anterior horn occurs in various conditions. It often happens as part of a diffuse myelitis, involving not only the anterior horn, but more or less completely the whole transverse section of the cord. In the acute atrophic paralysis of infants and adults (polio-myelitis anterior acuta, commonly called infantile paraly. sis), there is acute mye. litis, which is practically confined to the anterior horn of grey matter. In this disease the loss of faradaic excitability of the muscles is very constant and marked, In the chronic malady, how. ever, in which the same FIG. 4.

structure is affected (progressive muscular atrophy, pro topathic amyotrophy-Charcot), it is somewhat remarkable that, as Duchenne has shown, no decrease of faradaic excitability is to be noticed in the muscle. (This is no doubt the rule, but I have seen exceptions to it.) In the former case apparently a mass of ganglion-cells is suddenly, or at least very rapidly, subjected to a change which arrests their function, and which is followed by atrophy of more or less of their number, of which a varying proportion recovers. In the latter disease it would seem as though, in consequence of a slowly creeping lesion, the ganglion-cells atrophied gradually, leaving those of them which are spared unaltered, or at least with their function unimpaired, until they in their turn are invaded and destroyed. The relation of tendon-reflex to progressive muscular atrophy is a good deal complicated by the circumstance of there being at least two classes of this affection-the protopathic anyotrophy and the deuteropathic amyotrophy of Charcot. In typical cases the former is a very slowly progressive muscular atrophy, the gradual loss of power of the patient being in proportion to the extent to which the anterior horns of the cord have been invaded and the muscular system corre. spondingly atrophied. The latter begins with loss of power (paralysis) independent of muscular atrophy, anc is succeeded by a rapid muscular wasting of some or all of the muscles previously paralysed. It is this form which tends to extend into the medulla oblongata and to occasion death by destruction of the ganglion-cells essential to life, which are continuous upwards with those of the anterior horns of the spinal cord. The duration of this form is much shorter than that of the other. In the protopathic form the tendon. reflex may exist, to some extent at least, for a certain time, to be lost, however, when the atrophy has advanced, so that it is impossible to say that the behaviour of the tendon. reflex is at all characteristic. The break which causes its absence in such a case is in the anterior holn, as in Fig. 4.

The condition of the tendon-reflex in the deuteropathic form is extremely interesting. Before considering it, how. ever, I should like to draw attention to Fig. 5. In this diagram there is a rude representation of sclerosis of a lateral column of the spinal cord. In a previous diagram (Fig. 2) the position of sclerosis of the posterior root-zone was indicated. It was shown that if the afferent fibres from the tendon go, as we believe they do, with the sensory roots, they pass through the outer part of the posterior column, and consequently are exposed to a lesion which produces atrophic changes in them. The nervous are is therefore "cut" at this point, FIG. 5.

and that absence of tendon-reflex results, which is so conspicuous a feature of tabes dorsalis. The position of sclerosis of the lateral column, which we are now considering, is, it will be observed, altogether outside the nervous arc by which we 
suppose the stimulus from the tendon to the muscle to be transmitted. We should expect, therefore, to find that in sclerosis of a lateral column the tendon-reflex would not be absent. And that is the case. The tendon-reflex is never lost as a result of uncomplicated sclerosis of a lateral column.

So far from being lost, the tendon-reflex is indeed increased, frequently to a very surprising extent. Ankle clonus (also knee clonus-Erb, Gowers, Waller) may be, in these circumstances, produced not only (in the case of ankle clonus) by sudden dorsal flexion of the foot, ${ }^{3}$ but by the patient's own movements, as well as by light blows on various structures (bones, muscles, tendons), and sometimes even by the slight vibration produced by an accidental jar of his bedstead. This excitability to various kinds of stimulation is, however, by no means constant. One sees in certain sases of lateral sclerosis something like the converse of what $I$ have described as occurring in tabes. There is excessive tendon-reflex, knee clonus, as well as ankle clonus, yet the muscles concerned will not respond to a blow, and need a distinctly stronger faradaic current than in health to cause their contraction.

The question of prognosis is so mixed up with that of diagnosis in regard to the occurrence of ankle clonus, that the two may be considered together. The facts appear to show that in all cases the occurrence of increased tendonreflex in anything like a marked form signifies that from some cause the inhibitory influence of the higher centres is no longer being normally excited. It always means apparently that something has come in the way (at some point or other) of the natural propagation of nervous impulses from above. It is generally accepted that impulses from the highest centres (so-called volitional impulses) trave! to the ganglion-cells of the anterior horns by the anterolateral columns of the cord (pyramidal tracts). The fact that by what is commonly called "an effort of the will" we can control to a certain varying extent the reflex actions which are subserved in different parts of the cord would seem to show that in all probability the restraining influence which is continually and unconsciously being exerted over certain spinal reflexes in a state of health is conveyed by this same channel. ${ }^{4}$ Now it is when these (antero-lateral) columns are most extensively invaded by sclerosis, that is to say, when the resistance to the propagation downwards of nervous impulses from above is greatest, that we obtain the most marked ankle clonus. When there is a history pointing to myelitis at some portion of the cord, followed by a more or less pronounced rigidity of the limbs, with excess of tendon-reflex which has existed for some considerable time, say upwards of a year or two, we shall hardly be wrong in assuming that a change has taken place in the lateral columns which is likely to be permanent. And so also in reference to the like symptoms often consequent upon an attack of hemiplegia. An excess of reflex combined with more or less rigidity of the limbs, if it has lasted for months, may be considered as indicating a structural change in the lateral column which is unalterable. But this is by no means the case when the symptoms have not been of long standing. I have under my care at the present time in hospital a young woman who came in last June on account of paraplegia of ten months' duration, referable, as it seems, to a subacute myelitis in the dorsal region of the cord. On admission there was such a spastic condition of the legs that they contracted when handled or upon any attempt being made to flex the joints, which were stiff. There was great excess of patellar tendon-reflex, and ankle clonus was readily produced in a marked degree in each foot. The patient has gradually improved. She can stand and walk with very little help. There is no longer any marked spastic rigidity, and ankle clonus cannot now be obtained in either foot.

In hysteria the tendon-reflex is, so far as I have yet seen, usually increased, and in several cases $I$ have witnessed the occurrence of ankle clonus, the possibility of producing which has entirely, and in one case suddenly, ceased on the patient's recovery. I submit that this fact is an

3 I would refer to a lecture of mine in THE LANCET, Aug. 10th, 1878, for a detailed description of this phenomenon.

${ }_{4} \mathrm{Dr}$. Hughlings Jackson believes that the rigidity in hemiplegia results because by atrophy of the fibres of the lateral column of the cord "cerebral infuence" is taken off from the anterior horns, and thus the "cerebellar influence" upon them is no longer antagonised. On this prineiple, too, he would explain the increased tendon-reflex in cases of hemiplegia; mutatis mutandis, as regards lesions taking off the influence of the cerebellum.

extremely important one in reference to the causation of the phenomenon. There would seem to be but little doubt that in hysterical paraplegia the cerebral influence which is propagated downwards in health by the antero-lateral columns of the cord is more or less in abeyance. It may be suggested that the reason why an hysterical girl does not walk is because the nervous impulses from above, which ordinarily act upon the various spinal centres for progression, do not arrive there. The absence, then, of impulses dowuwards through the lateral columns seems to result in an effect upon the tendon-reflex which is very similar to that occasioned by the resistance to their propagation through the columns which is interposed by the structural change occurring in sclerosis.

It is necessary to remark, then, that the existence of ankle clonus cannot be considered conclusive evidence of a structural lesion of the spinal cord. It may be, to use a term which is generally understood, althongh scarcely capable of exact definition, "functional." I have never seen, however, in a case of hysteria an excessive degree of ankle clonus. The automatic flexion and extension movements of the foot will go on for perhaps a dozen times, becoming gradually weaker, and ceasing spontaneously.

The behaviour of the tendon-reflex in deuteropathic amyotrophy (lateral amyotrophic sclerosis) is important. Even when the disease is tolerably far advanced, and a good deal of a trophy of the muscles has taken place, it will be often found that the tendon-reflex not only remains, but may be in some excess. I have a patient now under observation who presents a typical example of the disease. He cannot stand without help, and the muscles of his thighs are not only much wasted, but their faradaic excitability is diminished, and they hang flabbily from his bones. Not only is the patellar tendon-reflex, however, in excess, but there is some ankle clonus occurring when he plants his foot in certain positions. I would suggest the following as an explanation of such cases as these (Fig. 6). So long as a certain number

FIG. 6 .

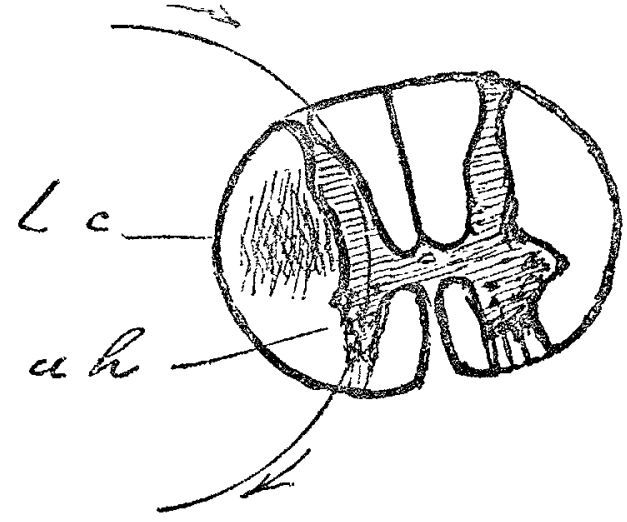

of ganglion-cells remain intact in the anterior horn the nervous are (for tendon-reflex) is complete. Now, if the lateral columns were sound, the inhibitory influence from above transmitted by them would be potent enough to hold the reflex, in its necessarily diminished strength, in complete restraint (as, for example, in infantile paralysis), and no response would therefore occur to a blow on the tendon. But the lateral column is diseased; the check is consequently removed, and the reflex manifests itself often with great energy. As a matter of course, however, it is not so strong as in that form of lateral sclerosis which is unattended by muscular atrophy.

In an earlier part of this paper $I$ have remarked that the response of the quadriceps to a blow on the patellar tendon "almost always signifies that the nervous arc is not seriously interrupted in that part of the spinal cord which gives origin to the lumbar plexus." In such a case as we are now considering (lateral sclerosis with muscular atrophy), it is evident that now and then the tendon-reflex may be apparently normal, and yet the spinal cord at the part which is concerned be affected with atroplyy of some of the ganglion-cells in the anterior horn. The defect in the reflex mechanism is, as it were, just balanced by the defect in the mecbanism which restrains reflex. It is necessary to bear this possibility in mind, in order to avoid an error, which, however, a little inquiry into the collateral circumstances will easily prevent. 